\title{
Obsolete Concept
}

National Cancer Institute

\section{Source}

National Cancer Institute. Obsolete Concept. NCI Thesaurus. Code C43634.

Concept is no longer considered correct, and should not be used except as encountered in mappings and old data. -- 2004. 